\title{
A Novel Reduction Strategy of Clarithromycin Resistance in Helicobacter pylori
}

\author{
Omid Tadjrobehkar ${ }^{1,2} ;$ Hamid Abdollahi ${ }^{1, *}$ \\ ${ }^{1}$ Department of Microbiology, Medical School, Kerman University of Medical Sciences, Kerman, IR Iran \\ ${ }^{2}$ School of Medicine, Zabol University of Medical Sciences, Zabol, IR Iran \\ ${ }^{*}$ Corresponding author: Hamid Abdollahi, Microbiology Department, Medical School, Kerman University of Medical Sciences, P. O. Box: 444, Kerman, IR Iran. Tel/Fax: +98-3413221665, \\ E-mail: ham.abdollahi@yahoo.com
}

Received: June 22, 2013; Revised: September 9, 2013; Accepted: September 11, 2013

\begin{abstract}
Background: Antibiotic resistance is a major therapeutic problem in patients infected with Helicobacter pylori. H. pylori clarithromycin resistant mutants have been evolved during antibiotic therapy, this is mainly due to $23 \mathrm{~s}$ rRNA point mutations.

Objectives: In the present study, we investigated anti-mutational features of four traditionally Iranian medicinal plants on three local isolated H.pylori strains.

Materials and Methods: In this study clarithromycin resistance was used as a mutation indicator. Frequencies of such mutations in the presence and absence of plant extracts were evaluated. Mutation incidence was evaluated by Luria Delbruck fluctuation assay.

Results: The mean mutation frequency in $H$. pylori isolates was $27 \times 10^{-9}$ which decreased at the presence of Mirtus communis, Teucrium polium, Achillea millefolium and Thymus vulgaris of plant extract, this amount was 97.4\%, 95.2\%, $63.7 \%$ and 19.6\% respectively. Moreover, A-to-G transition at 2143 position (A2143G) was detected by PCR-sequencing as major point mutation causing clarithromycin resistant mutants. Conclusions: The efficacy of these plant extracts in prohibiting resistance showed considerable results. This finding should be considered to use plant extracts with antibiotics to develop more effective eradication regimens.
\end{abstract}

Keywords:Medicinal Plants; Helicobacter pylori; Achillea millefolium; Teucrium polium; Mirtus communis; Clarithromycin; Mutation

\section{Background}

For the first time, Helicobacter pylori isolated from human gastric biopsies by Warren and Marshall in 1983 (1). Marshall reported that gastrointestinal disorders caused by self- ingestion of this bacterium (2). Today, this bacterium is known as the most common human pathogen affecting half of the world's population (3). The infections caused by H. pylori in human ranged from asymptomatic infections to symptomatic clinical presentations including acute or chronic gastritis, peptic ulcer diseases, gastric lymphoma and gastric adenocarcinoma (4). Eradication therapy is suggested for symptomatic patients. There are many different regimens used to treat $H$. pylori infections but, antibiotic resistance is the most important problems $H$. pylori eradication therapy $(3,5)$.

More than 60000 years ago, $H$. pylori colonized in human stomach and coevolved with humans since new human originated from his ancestors in Africa (6). Through the time H. pylori adapted to extraordinary acidic environment of gastric mucosa. The harsh environment of its niche enforces the bacterium to acquire particular mechanisms for rapid and effective adaptation to unfavorable conditions including presence of antimicrobial agents; as a result, $H$. pylori varied its gene pool. These variations were caused through point mutation, insertion, deletion or DNA exchange mechanisms (7).

There are some suspected mechanisms causing resistance against antibiotic agents but point mutation is accepted as the most important mechanism of antibiotic resistance in $H$. pylori (5). These sort of mutations occur at a very high frequency $\left(10^{-5}-10^{-8}\right)$ in $H$. pylori, which is much larger than many other bacteria such as Enterobacteriaceae (8). Therefore, anti-mutagenic agents could be beneficial in reducing the occurrence of resistance-conferring mutations (9). In this respect, medicinal plants could be used as the potential source.

\section{Objectives}

In this study, we tried to evaluate anti-mutagenic properties of some plant extracts on the occurrence of mutations conferring clarithromycin resistance in $H$. pylori.

\section{Materials and Methods}

\subsection{Plant Collection and Extraction}

In the present study, four herbs which are used in traditional Iranian medicine, including Teucrium polium (ger- 
mander), Achillea millefolium (yarrow), Thymus vulgaris (thyme) and Mirtus communis (myrtle) were purchased from a local medicinal herb shop. They were identified and confirmed by Professor G. H. Shahidi (Department of Botany, Faculty of Agriculture, Bahonar University, Kerman, Iran). The voucher numbers are kept at the Kerman University of Medical Sciences.

The seeds of M. communis, T. vulgaris leaves, flowers of $T$. polium and both leaves and flowers of A. millefolium were extracted as follows. The plant parts were washed, dried and grounded with a mechanical blender. Dry powders were soaked in extra pure ethanol (Merck, Germany) with solvent to sample ratio of 5:1 (v/w). After 5 days, each suspension filtered through Whatman filter paper No 5 (Whatman, England) and was concentrated, under reduced pressure. Each concentrated extract was evaporated to leave a solid pellet. The solid extracts were stored in dark at $-20^{\circ} \mathrm{C}$ until use. Before application the solid extracts were dissolved in dimethyl solfoxide (DMSO, Merck, Germany) to a final concentration of less than $1 \%$.

\subsection{Bacterial Isolation}

Three clarithromycin susceptible H. pylori isolates (K1, $\mathrm{K} 7$, and K11) were used in this study (see below). These isolates were obtained during a three-month-period from May to August 2011 from biopsy samples of patients referred to endoscopy unit of University hospital (Afzalipour, Kerman, Iran) in a previous study (10). All isolates were kept in brucella broth (BBL, USA) supplemented with $10 \%$ fetal bovine serum (Biochrom AG, Berlin, Germany) and $30 \%$ glycerol at $-70^{\circ} \mathrm{C}$.

\subsection{Selection of Claritromycin Susceptible Isolates}

Clarithromycin susceptibility was confirmed by inoculating a suspension of each isolate adjusted to a 3 of McFarland turbidity (approximately $9.0 \times 10^{8} \mathrm{CFU} . \mathrm{mL}^{-1}$ ) on Muller-Hinton agar (Merck, Germany) plates containing $10 \%$ fetal bovine serum and $1 \mu \mathrm{g}$ clarithromycin $\mathrm{mL}^{-1}$. Clarithromycin sensitive isolates were not able to grow at such concentration (11).

\subsection{Determination of Minimum Inhibitory Concen- tration}

Final concentrations of 62.5, 125, 250, 500 and 1000 $\mu \mathrm{g} . \mathrm{mL}^{-1}$ of the plant extracts were prepared for this purpose. Plant extracts were added to Muller-Hinton agar (Merck, Germany) enriched with 10\% fetal bovine serum to give the above mentioned final concentrations. Plates were spot inoculated by $2 \mu \mathrm{L}$ of a bacterial suspension adjusted to a turbidity 3 McFarland. The same plates without extract were also used as negative control. Incubation period was 3 days under micro aerobic atmosphere at $37^{\circ} \mathrm{C}(11)$.

The final concentrations of $0.03,0.06,0.125,0.25,0.5$ and $1 \mu \mathrm{g}$. $\mathrm{mL}^{-1}$ of clarithromycin were also used for de- termination the MIC of clarithromycin for each of these three isolates. Similar method and incubation condition were applied as above mentioned. Moreover, two concentrations (500 and $1000 \mu \mathrm{g} \cdot \mathrm{mL}^{-1}$ ) of each plant extract in combination with sub-inhibitory concentration (0.06 $\mu \mathrm{g} \mathrm{mL} \mathrm{m}^{-1}$ ) of clarithromycin in agar medium were used to evaluate the possible synergistic properties. Similar medium containing $0.06 \mu \mathrm{g}$. $\mathrm{mL}^{-1}$ clarithromycin without any plant extracts was also used as control.

\subsection{Determination of Mutation Frequency}

Mutation at 23s rRNA was introduced as the main cause of clarithromycin resistance in $H$. pylori. Therefore, in the present study we ignored clarithromycin. Mutation frequency was calculated using Luria Delbruck fluctuation assay by enumeration of observed mutant colonies grown from 20 parallel broth cultures in Brucella agar supplemented with $10 \%$ fetal bovine serum. Therefore, we prepared 25 independent tubes containing $1 \mathrm{~mL} \mathrm{Bru-}$ cella broth supplemented with 10\% fetal bovine serum. These tubes were inoculated with $10 \mu \mathrm{L}$ of a preliminary culture $\left(10^{4}-10^{5} \mathrm{CFU}\right.$. $\left.\mathrm{mL}^{-1}\right)$ of each isolate and were incubated under micro aerobic condition for 3 days at $37^{\circ} \mathrm{C}$. Five tubes from the above were used for enumeration of viable counts and $1 \mathrm{~mL}$ of each of the remaining 20 tubes was spread on agar media containing $1 \mu \mathrm{g}$. $\mathrm{mL}^{-1}$ clarithromycin. After a 3-day- incubation, the colonies on the agar plates were counted. Mutation frequency was calculated from the proportion of median number of counted mutant colonies in 20 tubes divided by the total viable counts $(12,13)$. A 50- $\mu \mathrm{L}-$ sample from preliminary culture was plated on agar media containing $1 \mu \mathrm{g}$. $\mathrm{mL}^{-1}$ clarithromycin to confirm no preexisting resistant mutants.

\subsection{Assessment of Mutation-Inhibiting Properties of Plant Extracts}

In order to determine any anti-mutational properties of the plant extracts, mutation frequency was calculated in the presence of five sub-inhibitory concentration of plant extracts $\left(62.5,125,250,500\right.$ and $\left.1000 \mu \mathrm{g} \cdot \mathrm{mL}^{-1}\right)$. The results were compared with the experimental condition in absence of plant extracts. All the experiments were performed in triplicates.

\subsection{DNA Extraction}

DNA extraction performed using Bioneer genomic kit (Bioneer, South Korea) and the DNA extracted from all three isolates. Furthermore, DNA extracted by same method from one resistant mutant colony related to each isolate that was picked up after mutation frequency assessment.

\subsection{PCR-Sequencing}

A pair of oligonucleotide primers (Table 1) obtained from the known sequences of 23s rRNA gene of a wild 
Tadjrobehkar O et al.

UA802 strain (GenBank accession No. U27270) were used to detect point mutations responsible for clarithromycin resistance. A 468 base pairs (bp) fragment corresponding to position 1811-2279 of the 23s rRNA gene was amplified in 35 cycles by a gradient thermal cycler (Biometra, Germany). PCR conditions were as follows, a primary denaturation at $94^{\circ} \mathrm{C}$ for 7 minutes followed by 1 minute denaturation at $94^{\circ} \mathrm{C}, 1$ minute annealing at $57^{\circ} \mathrm{C}, 1$ minute extension at $72^{\circ} \mathrm{C}$ and a final extension step for 5 minutes at $72^{\circ} \mathrm{C}$ as the end of amplification. The amplified fragments were sent to Macrogen Company (Gasan-dong, Geumchen-gu, Seoul, Korea) for sequencing. Point mutations were detected by comparing the sequences of the mutants (resistant colonies) with those of same fragment from 23s rRNA gene of a clarithromycin sensitive $H$. pylori strain UA802 in the GenBank, (accession No. U27270) (14).

\begin{tabular}{lcc}
\hline \multicolumn{3}{l}{ Table 1. Oligonucleotide Primers } \\
\hline Primers & Sequences $\left(\mathbf{5}^{-} \rightarrow \mathbf{3}^{\boldsymbol{}} \mathbf{)}\right.$ & Position \\
\hline HP-F & TAAGGTGTGCCACAGCGAT & $1811-1830$ \\
HP-R & CTAACAGAAACATCAAGGG & $\mathbf{2 2 7 9 - 2 2 6 0}$ \\
\hline
\end{tabular}

\section{Results}

\subsection{Extraction Yield}

Extraction yield (\% w/w) was calculated from the weight of final solid extract divided to the weight of the primary powdered plant parts. Our method have an extraction yield of 5\% for M. communis, $7 \%$ T. vulgaris and A. millefolium to $9 \%$ for T. polium.

\subsection{Minimal Inhibitory Concentration Values}

The growth of all three isolates were inhibited at the concentration of $0.125 \mu \mathrm{g}$. $\mathrm{mL}^{-1}$ of clarithromycin and above. MIC of clarithromycin on mutated resistant colonies raised to more than $16 \mu \mathrm{g} \cdot \mathrm{mL}^{-1}$. Anti-Helicobacter properties of the plant extracts were evaluated at five different concentrations (62.5, 125, 250, 500 and 1000 $\left.\mu \mathrm{g} \cdot \mathrm{mL}^{-1}\right)$. All of the applied extracts, except M. communis in the above mentioned concentration had shown no effect on the tested bacterial isolates. Therefore, it is expected that MIC of these extracts is more than $1000 \mu \mathrm{g} . \mathrm{mL}^{-1}$. The MIC against M. communis was $1000 \mu \mathrm{g} \mathrm{mL}^{-1}$. There was not any observed synergism between $M$. communis concentration less than $1000 \mu \mathrm{g} . \mathrm{mL}^{-1}$ and $0.06 \mu \mathrm{g}$. $\mathrm{mL}^{-1}$ of clarithromycin.

\subsection{Point Mutation Conferring Resistance to Clar- ithromycin}

DNA sequences of a 468-bp-fragment of the 23s rRNA gene from preliminary isolated bacteria (K1, K7, K11) compared with DNA sequences of clarithromycin sensitive wild UA802 strain. The results were completely identical; therefore preliminary isolated bacteria were preliminary confirmed as clarithromycin susceptible. A-to-G transition at 2143 position (A2143G) was detected as major point mutation in the sequences associated with the resistant mutant colonies incorporated to each of isolates.

\subsection{Mutation Frequency of Clarithromycin Resis- tance}

Mutation frequency was separately calculated for each isolate. Some minor differences between values were observed. The overall mean value was calculated to be $27 \times 10^{-9}$.

\subsection{Mutation Frequency Values Applying the Plant Extracts}

Results obtained from these experiments showed that at only three concentrations $\left(250,500\right.$ and $\left.1000 \mu \mathrm{g} \mathrm{mL}^{-1}\right)$, the plant extract were effective in reducing the mutation frequency compared to the results obtained at the absence of plant extracts. Data collected after application of different concentrations of plant extracts were very similar (data not showed); therefore, we choose the minimal effective concentration at $250 \mu \mathrm{g} \mathrm{mL}^{-1}$ for the rest of experiments. Our findings showed a significant reduction in the mutation frequency value after application of extracts of M.communis, T. polium and A. millefolium, but, $T$. vulgaris extract was not as effective as the others. All three isolate were affected by these four plant extract with a minor differences.

The mean mutation frequency in the presence of extracts obtained from $T$. vulgaris and A. millefolium has been reduced to $21.7 \times 10^{-9}$ and $9.8 \times 10^{-9}$ respectively. In duplicated application of M. communis and T. polium extracts, we have not observed any mutant colony in agar media, though, bacterial cell suspension contained. $10^{9}$ CFU.mL ${ }^{-1}$. Therefore, bacterial suspension containing $10^{11}$ CFU.mL ${ }^{-1}$ was used in these cases. After inoculation of this bacterial suspension on the agar media containing $M$. communis and T. polium extracts the mean mutation frequency values were $0.7 \times 10^{-9}$ and $1.3 \times 10^{-9}$ respectively.

\section{Discussion}

Successful eradication of $H$. pylori usually resulted in resolving the symptoms of its infection; moreover, it may have resulted in preventing the possible gastric cancer. Different eradication regimens have been developed in this respect. In the recent decades, the clarithromycinbased triple therapy was used as the recommended firstline empirical treatment of $H$. pylori infections $(3,5)$. Antibiotic resistance, especially clarithromycin resistance, is significantly increased all over the world. Therefore it is no longer recommended to follow empirical therapy of $H$. pylori infections in areas with high resistance rates (3, $15,16)$. Point mutations in 23s rRNA gene was described as the main mechanism of clarithromycin resistance in 
H.pylori (17). The inhibition of these resistance-conferring mutations could serve as a novel strategy to overcome the antibiotic resistance issue. In recent years, a large number of literature reported the suggested anti-mutagenic agents in the medicinal plants (18-20).

Mutation is a complex process changing a genotype but not necessarily the phenotype. Therefore, the number of mutant colonies are significantly lower than the genotypically changed bacteria $(11,21,22)$. We tracked the changes in the occurrence of mutation events which took place upon application of medicinal plants by means of "Mutation Frequency". Mutation frequency shows the actual consequence of this intervention, because only the favorable mutation resulted in the appearance of an antibiotic resistant phenotype are detected.

The results obtained by comparing the mean mutation frequency values in the presence and absence of plant extracts. It is shown that there are reductions in the mean mutation frequency values after application of $M$. communis, T. polium, A. millefolium and T. vulgaris this amounts were $97.4 \%, 95.2 \%, 63.7 \%$ and $19.6 \%$, respectively. This was an interesting outcome which could explain the existence of certain mutation preventative potentials in these plant extracts. Our results reveal that the ethanolic extract from T. vulgaris was not so effective in reducing the mutation frequency of clarithromycin resistance. This is in contrast with the results of Wei and Shibamoto (23). Such differences may be attributed to essential oil effects in their experiments rather than the ethanolic extract which was used in our experiments. Aidi Wannes et al. reported weak anti-oxidant and probably anti-mutational activities of essential oils extracted from $M$. communis (24). They used essential oils obtained from leaf; stem and flower of this herb but ethanolic extract of the seeds was used in our experiment which showed its potent anti-mutational activity. These results demonstrated that the efficacy of ethanolic extracts is higher than that of essential oils. This finding was also supported by Aidi Wannes et al. (24). The results from previous studies reporting the anti-mutagenic activity and free radical-scavenging properties of these herbs supported our findings $(18,19,25-29)$. The reduction effect of plant extracts on antibiotic resistance may justifies the usage of these medicinal plant extracts.

Some appropriate experiments under in vivo condition on animal models or human volunteers are needed to confirm these findings. In this respect, the medicinal plant derived substances in comparison to the chemical agents could obtain more patients compliance and ease in designing of clinical trials in human volunteers. The extracts obtained from the tested herbs, beside antimutational effects had reported anti-inflammatory and protective effect on gastric mucosal layer (30). Such valuable findings confirmed the beneficial use of those in traditional herbal medicine for treatment of gastrointestinal discomforts especially those caused by $H$. pylori. The accumulative results obtained from such investigations could lead to the development of more effective eradica- tion regimens containing medicinal plants in combination with antibiotics. This may even be more beneficial if the actual effective ingredients from such plant extracts are recognized and the most suitable is to be added to the present antibiotic regimens.

\section{Authors' Contributions}

Omid Tadjrobehkar developed the primary idea and research protocol. Both of authors equally contributed in preparation of the manuscript.

\section{Funding/Support}

Economical resources were developed by research committee of Kerman University of Medical Sciences, Kerman, Iran (grant No. 90/246).

\section{References}

1. Unidentified curved bacilli on gastric epithelium in active chronic gastritis. Lancet. 1983;1(8336):1273-5.

2. Marshall BJ, Armstrong JA, McGechie DB, Glancy RJ. Attempt to fulfil Koch's postulates for pyloric Campylobacter. Med J Aust. 1985;142(8):436-9.

3. Malfertheiner P, Megraud F, O'Morain CA, Atherton J, Axon AT, Bazzoli F, et al. Management of Helicobacter pylori infection--the Maastricht IV/Florence Consensus Report. Gut. 2012;61(5):646-64.

4. McColl KE. Clinical practice. Helicobacter pylori infection. N Engl JMed. 2010;362(17):1597-604.

5. Wu W, Yang Y, Sun G. Recent Insights into Antibiotic Resistance in Helicobacter pylori Eradication. Gastroenterol Res Pract. 2012;2012:723183.

6. Atherton JC, Blaser MJ. Coadaptation of Helicobacter pylori and humans: ancient history, modern implications. J Clin Invest. 2009;119(9):2475-87.

7. de Reuse H, Bereswill S. Ten years after the first Helicobacter pylori genome: comparative and functional genomics provide new insights in the variability and adaptability of a persistent pathogen. FEMS Immunol Med Microbiol. 2007;50(2):165-76.

8. Kraft C, Suerbaum S. Mutation and recombination in Helicobacter pylori: mechanisms and role in generating strain diversity. Int J Med Microbiol. 2005;295(5):299-305.

9. Cirz RT, Chin JK, Andes DR, de Crecy-Lagard V, Craig WA, Romesberg FE. Inhibition of mutation and combating the evolution of antibiotic resistance. PLoS Biol. 2005;3(6).

10. Abdollahi H, Tadjrobehkar O. The role of different sugars, amino acids and few other substances in chemotaxis directed motility of helicobacter pylori. Iran J Basic Med Sci. 2012;15(3):787-94.

11. Bjorkholm B, Sjolund M, Falk PG, Berg OG, Engstrand L, Andersson DI. Mutation frequency and biological cost of antibiotic resistance in Helicobacter pylori. Proc Natl Acad Sci U S A. 2001;98(25):14607-12.

12. Rosche WA, Foster PL. Determining mutation rates in bacterial populations. Methods. 2000;20(1):4-17.

13. Wang G, Wilson TJ, Jiang Q, Taylor DE. Spontaneous mutations that confer antibiotic resistance in Helicobacter pylori. Antimicrob Agents Chemother. 2001;45(3):727-33.

14. Taylor DE, Ge Z, Purych D, Lo T, Hiratsuka K. Cloning and sequence analysis of two copies of a 23S rRNA gene from Helicobacter pylori and association of clarithromycin resistance with $23 \mathrm{~S}$ rRNA mutations. Antimicrob Agents Chemother. 1997;41(12):2621-8.

15. De Francesco V, Giorgio F, Hassan C, Manes G, Vannella L, Panella C, et al. Worldwide H. pylori antibiotic resistance: a systematic review. J Gastrointestin Liver Dis. 2010;19(4):409-14.

16. Megraud F, Coenen S, Versporten A, Kist M, Lopez-Brea M, Hirschl AM, et al. Helicobacter pylori resistance to antibiotics in Europe and its relationship to antibiotic consumption. Gut. 2013;62(1):34-42. 


\section{Tadjrobehkar O et al.}

17. Versalovic J, Shortridge D, Kibler K, Griffy MV, Beyer J, Flamm RK, et al. Mutations in 23S rRNA are associated with clarithromycin resistance in Helicobacter pylori. Antimicrob Agents Chemother. 1996;40(2):477-80.

18. Kumar RV, Venkatrajireddy G, Bikshapathi T, Reddy MK. Antioxidant-the maximum expressed activity among 63 medicinal plants. J Phyto Pharmacol. 2012;1(5):1-13.

19. Milosevic-Djordjevic O, Stosic I, Stankovic M, Grujicic D. Comparative study of genotoxicity and antimutagenicity of methanolic extracts from Teucrium chamaedrys and Teucrium montanum in human lymphocytes using micronucleus assay. Cytotechnology. 2013;65(5):863-9.

20. Sibanda T, Okoh AI. The challenges of overcoming antibiotic resistance: Plant extracts as potential sources of antimicrobial and resistance modifying agents. Afr JBiotechnol. 2007;6(25):2886-96.

21. Andersson DI. The biological cost of mutational antibiotic resistance: any practical conclusions? Curr Opin Microbiol. 2006;9(5):461-5.

22. De Francesco V, Zullo A, Ierardi E, Giorgio F, Perna F, Hassan C, et al. Phenotypic and genotypic Helicobacter pylori clarithromycin resistance and therapeutic outcome: benefits and limits. J Antimicrob Chemother. 2010;65(2):327-32.

23. Wei A, Shibamoto T. Antioxidant/lipoxygenase inhibitory activities and chemical compositions of selected essential oils. J Agric
Food Chem. 2010;58(12):7218-25.

24. Aidi Wannes W, Mhamdi B, Sriti J, Ben Jemia M, Ouchikh O, Hamdaoui $\mathrm{G}$, et al. Antioxidant activities of the essential oils and methanol extracts from myrtle (Myrtus communis var. italica L.) leaf, stem and flower. Food Chem Toxicol. 2010;48(5):1362-70.

25. Hayder N, Abdelwahed A, Kilani S, Ammar RB, Mahmoud A, Ghedira K, et al. Anti-genotoxic and free-radical scavenging activities of extracts from (Tunisian) Myrtus communis. Mutat Res. 2004;564(1):89-95.

26. Mahady GB, Pendland SL, Stoia A, Hamill FA, Fabricant D, Dietz $\mathrm{BM}$, et al. In vitro susceptibility of Helicobacter pylori to botanical extracts used traditionally for the treatment of gastrointestinal disorders. Phytother Res. 2005;19(11):988-91.

27. Meena AK, Bansal P, Kumar S. Plants-herbal wealth as a potential source of ayurvedic drugs. Asian J Tradi Med. 2009;4(4):152-70

28. Miguel MG. Antioxidant and anti-inflammatory activities of essential oils: a short review. Molecules. 2010;15(12):9252-87.

29. Wojdylo A, Oszmianski J, Czemerys R. Antioxidant activity and phenolic compounds in 32 selected herbs. Food Chem. 2007;105(3):940-9.

30. Zaidi SF, Muhammad JS, Shahryar S, Usmanghani K, Gilani AH, Jafri W, et al. Anti-inflammatory and cytoprotective effects of selected Pakistani medicinal plants in Helicobacter pylori-infected gastric epithelial cells. J Ethnopharmacol. 2012;141(1):403-10. 